\title{
Crown to implant ratio in palatal molar implants.
}

Piotr Wychowański ${ }^{1}$, Paweł Nieckulaa ${ }^{1}$, Artur Rasiński ${ }^{1}$, Bartłomiej Iwańczyk ${ }^{1}$, Zbigniew Kucharski², Andrzej Wojtowicz ${ }^{1}$

${ }^{1}$ Department of Oral Surgery, Medical University of Warsaw, Warsaw, Poland

2Department of Prosthetic Dentistry, Medical University of Warsaw, Poland

Background I. The influence of the crown to implant ratio on the longevity of implant restorations

II. The fate of the residual bone after the molar extraction in the maxilla

Sinus lift
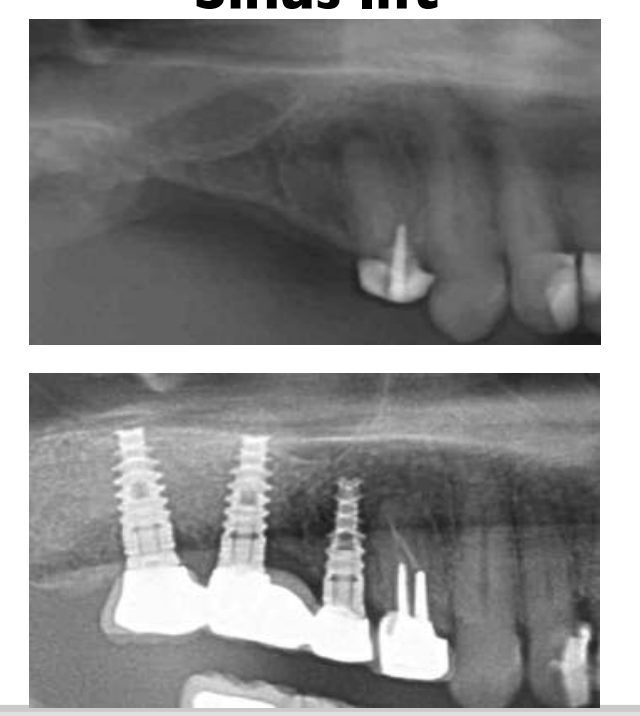

Crown to implant ratio is essential factor for long - term survival of dental implants.

The extractions of permanent molars in maxilla are followed

by alveolar bone resorption and pneumatisation of maxillary sinus. ${ }^{2}$

Sinus floor elevation procedures allows dental implant placements in the cases of alveolar bone deficiency ${ }^{3}$

The positioning of the implants in these cases are frequently unfavourable due to the depressed alveolar ridge level 4

Immediate implants inserted in the fresh palatal socket of the extracted first or second maxillary molar tooth prevents alveolar ridge resorption ${ }^{5}$
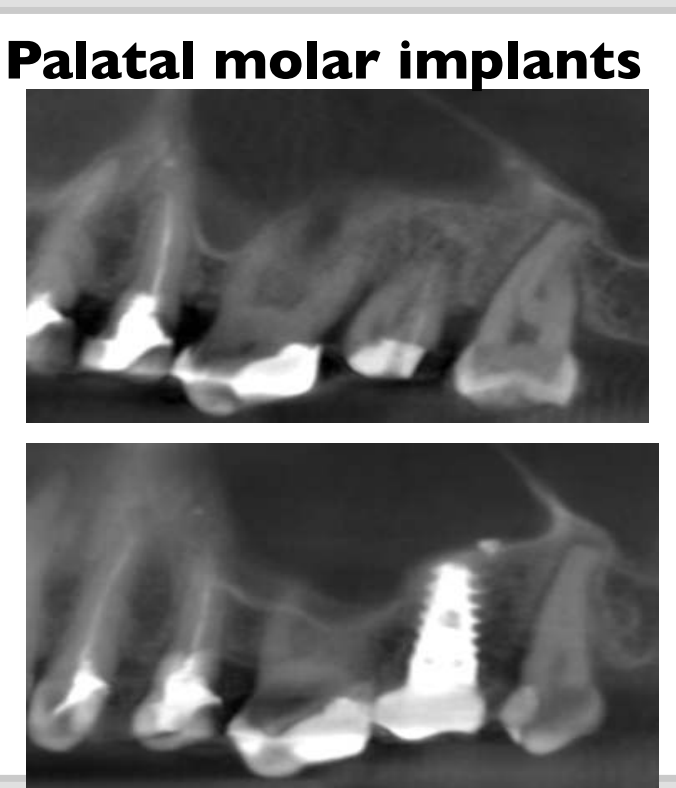

Aim/Hypothesis: Palatal molar implants show more favourable crown to implant ratio than implants inserted after sinus lift procedure
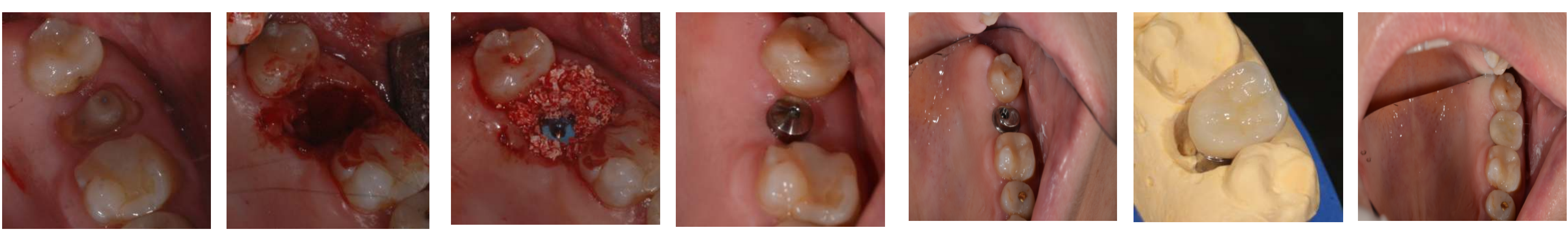

Materials and methods
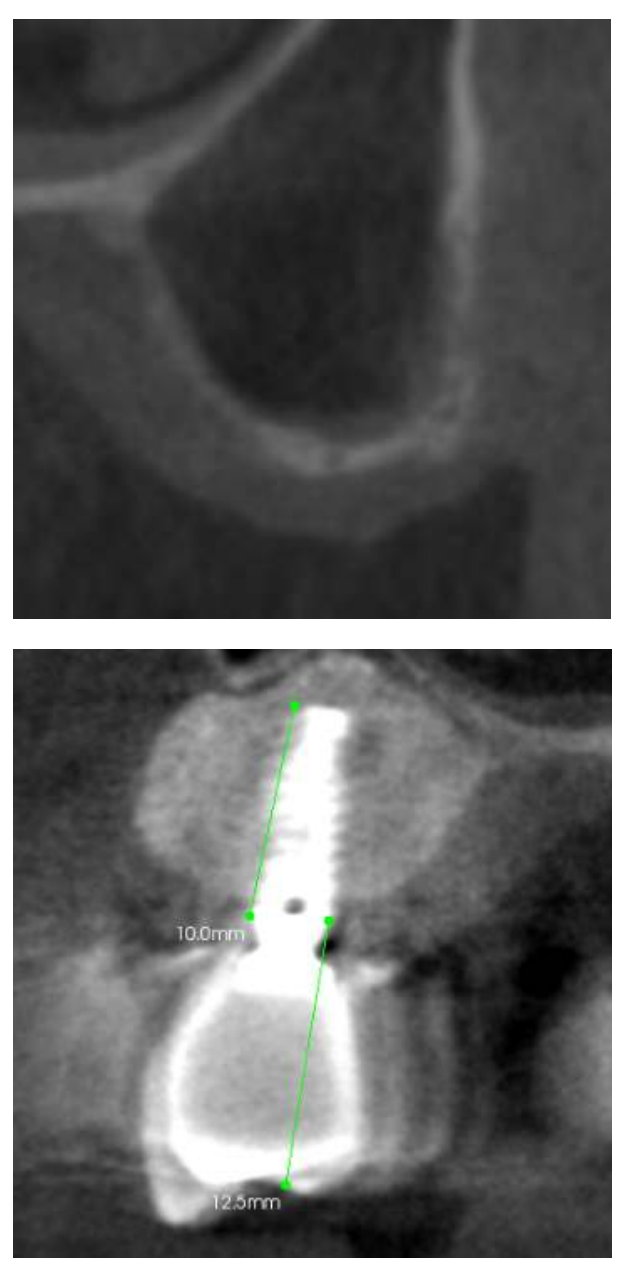

The crown to implant ratio of the implants inserted in molar region of the maxilla was measured on CBCT scans.

All implants were restored with cemented crowns or bridges.

The examination group consists of 61 implants inserted in 52 patients.

All the implants were inserted immediately after extraction of the first or the second upper molar. The palatal fresh socket served as a implant bed. Bone deficiencies were filled with xenograft bovine material. Surgical cement was used in the first week of healing to prevent blood cloth and augmentation material loss. The implants were restored with the cemented crowns 6 months after implantation.

The control group consists of 100 implants inserted in 52 patients. All the implants were inserted in the molar region of maxilla after sinus floor elevation procedure. Bovine xenograft material was used in all of the cases in the control group. All implants were restored 6 months after implantation with crowns or bridges.

The length of the used implants and the height of the used prosthetic superstructures were measured on CBCT scans. Crown to implant ratio was calculated in all the cases.
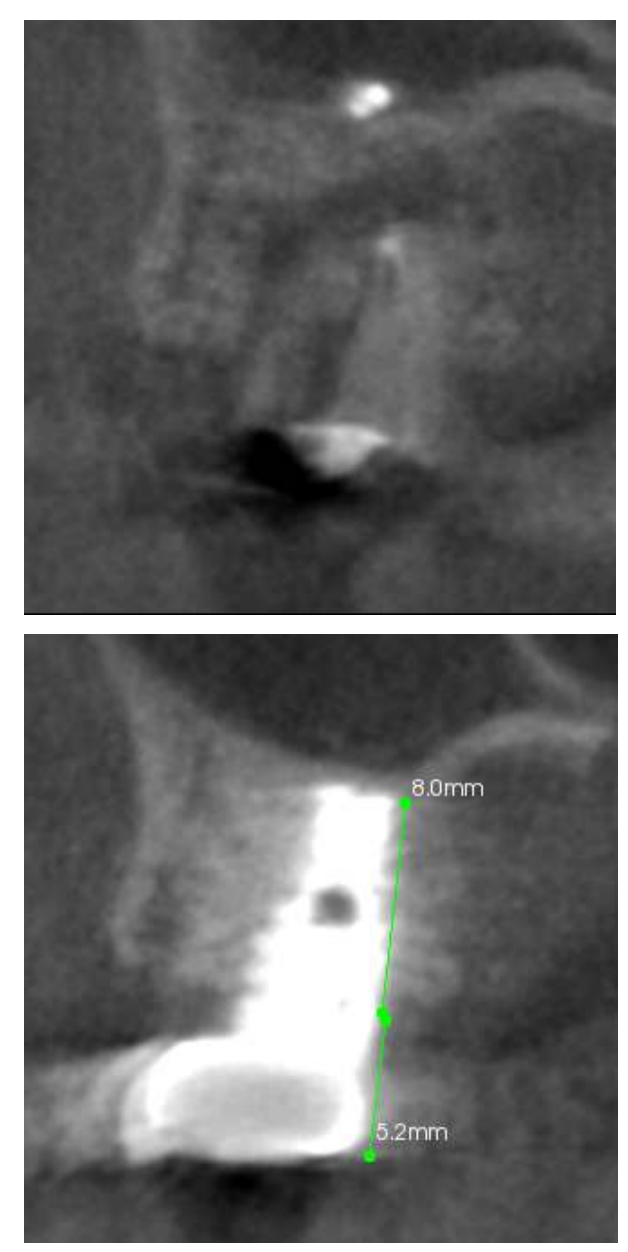

\section{Results}

I. All the 61 implants inserted into the palatal fresh socket after the extraction of the first or the second upper molar teeth obtained good primary stability, successfully healed and was restored after 6 months.

2. The implants inserted in this novel immediate technique was revealed no clinical problems after 24 months of follow up.

3. The mean crown to implant ratio in the experimental group was 0,696

4. The mean crown to implant ratio in the control group was I,I03

5. The experimental group revealed statistically significant $p<0,005$ favourable crown to implant ratio in comparison with the control group.

\section{Conclusions}

(1) Palatal molar implants shows favourable crown to implant ratio if compare to the implants restored after conventional sinus floor elevation procedure.

(2) Favourable crown to implant ratio is essential for occlusal stress distribution around the implant. It may result in better longevity of the implants inserted immediately after upper molar teeth extractions if compare to the staged approach.

(3) The study require further study and longer follow ups to confirm this results.

\section{REFERENCES:}

1. Shan-Pao S, Ik-Sang M, Kwang-Ho P, Dong-Won L. Effects of Crown to Implant Ratio and Anatomical Crown Lenght on Clinical Conditions in a Single implant:A Retrospective Cohort Study. Clin. Impl. Dent.And Related Research,August 2015,Vo. 17 (4), PP

$724-731$.

2. Sharan A, David Madjar D. Maxillary Sinus Pneumatization Following Extractions:A Radiographic Study. Int J Oral Maxillofac Implants 2008;23:48-56.

3. Pjetursson BE, Tan WC, Zwahlen M, Lang NP.A systematic review of the success of sinus floor elevation and survival of implants inserted in combination with sinus floor elevation. Part I: Lateral approach.J Clin Periodontol 2008;35:216-240.

4.Tan WC, Lang NP, Zwahlen M, Pjetursson BE.A systematic review of the success of sinus floor elevation and survival of implants inserted in combination with sinus floor elevation. Part Il:Transalveolar technique.J Clin Periodontol 2008;35:24I-254.

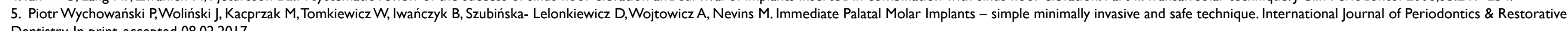

